\title{
A New Control Method for Second-Order Multiple Models Control System Based on Global Sliding Mode
}

\author{
Dan-xu Zhang $\mathbb{D}^{\mathbb{D}}{ }^{1}$ Yang-wang Fang $\mathbb{D}^{1},{ }^{1}$ Peng-fei Yang $\mathbb{D}^{\mathbb{2}},{ }^{2}$ You-li $W u,{ }^{1}$ and Tong-xin Liu ${ }^{1}$ \\ ${ }^{1}$ School of Aeronautics Engineering, Air Force Engineering University, Xian, China \\ ${ }^{2}$ Research Center for Evaluation and Argumentation, Academy of Military Sciences, Beijing 100091, China \\ Correspondence should be addressed to Dan-xu Zhang; zdx1989818@163.com
}

Received 11 January 2018; Revised 23 May 2018; Accepted 28 June 2018; Published 30 July 2018

Academic Editor: Andrzej Swierniak

Copyright (c) 2018 Dan-xu Zhang et al. This is an open access article distributed under the Creative Commons Attribution License, which permits unrestricted use, distribution, and reproduction in any medium, provided the original work is properly cited.

\begin{abstract}
This paper proposed a finite time convergence global sliding mode control scheme for the second-order multiple models control system. Firstly, the global sliding surface without reaching law for a single model control system is designed and the tracking error finite time convergence and global stability are proved. Secondly, we generalize the above scheme to the second-order multimodel control system and obtain the global sliding mode control law. Then, the convergent and stable performances of the closed-loop control system with multimodel controllers are proved. Finally, a simulation example shows that the proposed control scheme is more effective and useful compared with the traditional sliding mode control scheme.
\end{abstract}

\section{Introduction}

Many control systems work in complicated conditions in reality and are faced with the uncertainty brought by parameter variety $[1,2]$. For instance, the controller of hypersonic vehicle works in complicated environment caused by fast speed [3-6]. Furthermore, the controller demands highly robustness to uncertainty due to the varying parameters brought by large envelope, hypersonic velocity, and strong coupling [7-9]. Approximating control system via multimodel is one scheme to reduce uncertainty. Many control schemes, such as adaptive control [10], variable structure control [11], and robust control [12] were applied to the multimodel system.

Sliding mode control (SMC) [13-17] has gained widely spread due to its characteristics of robustness to uncertainties. However, the chattering problem and matching condition (the relative degree of the sliding system must be 1) limit its applications in practice [18].

In order to attenuate the chattering effect, a high-order sliding mode control (HOSMC) [19] scheme was proposed and the controller obtained less chattering and finite time convergence performances. Nevertheless, designing the controller based on HOSMC for multimodel is a heavy work.
Particularly, slow reaction and convergence, poor transient response, and unstable system behaviours always occur at the switching instant between submodels due to the parameters' boundary changes or large external disturbance [20]. Thus, the robustness to the switching instant is more important for the overall closed-loop control system. However, the robustness of SMC and HOSMC is meaningful only in the sliding phase [18], which means that the reaching phase reduces the robust performance of the closed-loop system [21].

Choi Seung-Bok [22] presented a time-varying sliding surface for second-order system and reduces the reaching phase. Chang and Hurmuzlu [23] improved the time-varying sliding surface and designed a new sliding control scheme without reaching phase. The global sliding mode control (GSMC) [24-28] has been proposed and ensures the existence of sliding mode throughout the entire control response. Furthermore, for multimodel control problem, GSMC can ensure that state variables move along the surface and reduce the convergence time at switching instant due to eliminating reaching phase. It is robust to matched perturbations in both sliding phase and switching instant. Thus, the GSMC has advantages on finite time convergence for multimodel control problem. 
In this paper, the GSMC is introduced to design a controller for the second-order multimodel system. The paper is organized as follows: Section 2 states the multimodel control problem. In Section 3, the GSMC is derived and the closed-loop control system finite time convergence and global stability in both single model and multimodel are proved. The performance of GSMC controller is verified via computer simulations compared with SMC in Section 4.

\section{Problem Statement}

Suppose that a nonlinear system can be linearization at a series of feature points as second-order multimodel systems with uncertainties and one of the submodels is described by [30]

$$
\ddot{x}+F_{i}(t) \dot{x}=G_{i}(t) u_{i}+d_{i}(t)
$$

Then the second-order systems constitute the multimodel to approximate the original nonlinear system and can be expressed as

$$
\begin{gathered}
M_{1}: \ddot{x}+F_{1}(t) \dot{x}=G_{1}(t) u_{1}+d_{1}(t) \\
M_{2}: \ddot{x}+F_{2}(t) \dot{x}=G_{2}(t) u_{2}+d_{2}(t) \\
\vdots \\
M_{i}: \ddot{x}+F_{i}(t) \dot{x}=G_{i}(t) u_{i}+d_{i}(t) \\
\vdots \\
M_{N}: \ddot{x}+F_{N}(t) \dot{x}=G_{N}(t) u_{N}+d_{N}(t), \\
\left\|d_{i}(t)\right\| \leq D
\end{gathered}
$$

where $x=\left[x_{1}, x_{2}\right]^{T} \in \mathbf{R}^{2}, u \in \mathbf{R}, F_{i}(t)$ and $G_{i}(t)$ are continuous sufficiently smooth functions, respectively, and $G_{i}(t) \neq 0 ; d_{i}(t)$ is the disturbance with boundary $\left\|d_{i}(t)\right\| \leq D$.

The purpose is to design a valid control scheme that can achieve fast stable control performance for each model $S_{i}$ and be robust to the switching instant.

Particularly, the switching between submodels discussed here is in order. For instance, the flight control system of aircraft can be approximated by some submodels that divided by height range and when the height changes from the range of one submodel to another, the submodel switches. As height changes continuously, the multimodel switches in order. Thus, it is assumed that the multimodel $M$ switches in order with state $x$ changing and the switching order is $M_{i} \longrightarrow M_{i+1}$. Furthermore, the multimodel can be rewritten as

$$
\begin{aligned}
& M_{1}: \ddot{x}+F_{1}(t) \dot{x}=G_{1}(t) u_{1}+ d_{1}(t), \\
& \text { if } x_{1}^{\text {initial }} \leq x<x_{1}^{\text {terminal }} \\
& M_{2}: \ddot{x}+F_{2}(t) \dot{x}=G_{2}(t) u_{2}+d_{2}(t), \\
& \\
& \text { if } x_{2}^{\text {initial }} \leq x<x_{2}^{\text {terminal }}
\end{aligned}
$$

$$
\begin{gathered}
M_{i}: \ddot{x}+F_{i}(t) \dot{x}=G_{i}(t) u_{i}+d_{i}(t), \\
\quad \text { if } x_{i}^{\text {initial }} \leq x<x_{i}^{\text {terminal }} \\
\vdots \\
M_{N}: \ddot{x}+F_{N}(t) \dot{x}=G_{N}(t) u_{N}+d_{N}(t), \\
\text { if } x_{N}^{\text {initial }} \leq x<x_{N}^{\text {terminal }}
\end{gathered}
$$

where $x_{i}^{\text {initial }}$ and $x_{i}^{\text {terminal }}$ are the initial and terminal state value of the $i^{\text {th }}$ submodel corresponding range.

\section{Global Sliding Mode Control}

3.1. Single Model Control. Considering a single model $M$ expressed as

$$
\ddot{x}+F(t) \dot{x}=G(t) u+d(t), \quad\|d(t)\| \leq D
$$

we design the global sliding surface as

$$
s=\dot{e}+c e-f(t)
$$

in which $e=x-x_{c}$ is the track error. $x_{c}$ is the reference command signal. $f(t)=[\dot{x}(0)+c x(0)] \exp (-k t)$ is the forcing function and is 1st-order differentiable with $\lim _{t \rightarrow \infty} f(t)=0$. Moreover, $c=$ const. $>0, k=$ const. $>0$.

Obviously, due to the additional time-varying term $f(t)$ in (5), the global sliding surface can ensure that the state of the closed-loop control system moves along the sliding surface from the beginning. Thus, there is no need for reaching law.

3.1.1. Lyapunov Stability Analysis. According to the equivalent control method, let $\dot{s}=0$ and, then, we obtain the following equation:

$$
\dot{s}=\ddot{e}+c \dot{e}-\dot{f}(t)=\ddot{x}-\ddot{x}_{c}+c\left(\dot{x}-\dot{x}_{c}\right)-\dot{f}(t)=0
$$

Substitute (4) into (6), the control law is solved as

$$
u=-\frac{[-F \dot{x}+c \dot{x}-\dot{f}+R+(\varepsilon+D) \operatorname{sign}(s)]}{G}
$$

where $R=-\ddot{x}_{c}-c \dot{x}_{c}, \varepsilon=$ const. $>0$ and $\varepsilon>-2 D$. $D$ is the supremum of $d(t)$. Additionally, without ambiguity we take $F, G$, and $f$ as brief form for $F(t), G(t)$, and $f(t)$, respectively.

To prove the stability of the control scheme, we define the Lyapunov function as $V=(1 / 2) s^{2}$ and the derivative $\dot{V}$ is

$$
\begin{aligned}
\dot{V} & =s \dot{s}=s(\ddot{e}+c \dot{e}-\dot{f})=s\left(\ddot{x}-\ddot{x}_{c}+c\left(\dot{x}-\dot{x}_{c}\right)-\dot{f}\right) \\
& =s\left(-\ddot{x}_{c}-c \dot{x}_{c}+c \dot{x}-\dot{f}-F \dot{x}+G u+d\right)=s\{R \\
& +c \dot{x}-\dot{f}-F \dot{x}+d \\
& \left.+G\left\{-G^{-1}[-F \dot{x}+c \dot{x}-\dot{f}+R+(\varepsilon+D) \operatorname{sign}(s)]\right\}\right\} \\
& =s(d-(\varepsilon+D) \operatorname{sign}(s))=d s-D|s|-\varepsilon|s|
\end{aligned}
$$


If $s>0, \dot{V}=s \dot{s}=d s-D s-\varepsilon s$. The derivative satisfies $\dot{V}<0$ because of $|d|<D$ and $\varepsilon>0$. If $s<0, \dot{V}=s \dot{s}=$ $d s+D s+\varepsilon s$, and with the same parameter conditions the derivative satisfies $\dot{V}<0$. Therefore, the closed-loop control system based on GSMC is globally stable.

3.1.2. Finite Time Convergence Analysis. Consider the sliding mode system (5) and rewrite it as

$$
s(e(t))=\dot{e}(t)+c e(t)-f(t)
$$

where $f(t)=[\dot{e}(0)+c e(0)] \exp (-k t)$. To prove that the sliding mode system (9) is convergent in finite time, we can solve the equation $s=0$ to obtain the convergence time $T_{c}$.

Let $L=[\dot{e}(0)+c e(0)]$; the differential equation about $e(t)$ is

$$
\dot{e}+c e-L \exp (-k t)=0
$$

According to [31], it is a kind of Bernoulli equation, and $e(t)$ is given by

$$
e(t)=\frac{L}{c-k} \exp (-k t)+m \exp (-c t)
$$

where $m$ is an arbitrary constant.

To obtain the convergence time $T_{c}$, take the natural logarithm of both sides in (11) and $T_{c}$ is

$$
T_{c}=\frac{1}{k-c} \ln \left(\frac{L}{m(k-c)}\right)
$$

Obviously, the parameters in (12) must satisfy the following constraints: $c \neq k, L \neq 0, m \neq 0$, and $L / m(k-c)>1$.

In particular, $T_{c}$ is closely related to the parameters $k$, $c$, and $m . T_{c}$ reduces with the increasing of $k-c$. What is more, the value of $\ln (L / m(k-c))$ is not large due to the character of the function $y=\ln x$, and, for example, $\ln 10^{10} \approx$ 23.03. Therefore, with reasonable design value of $k-c$, the convergence time $T_{c}$ is finite.

3.2. Multiple Models Control. In the following section, we will discuss multimodel controller design based on the GSMC.
Considering the multimodel $M:\left[M_{1}, M_{2}, \ldots, M_{N}\right]$, firstly we design sliding surfaces $s_{1}, s_{2}, \ldots, s_{i} \ldots, s_{N}$ for each model $M_{i}$ as follows:

$$
\begin{gathered}
s_{1}=\dot{e}+c_{1} e-L_{1} \exp \left[-k_{1}\left(t-t_{1}\right)\right] \\
s_{2}=\dot{e}+c_{2} e-L_{2} \exp \left[-k_{2}\left(t-t_{2}\right)\right] \\
\vdots \\
s_{i}=\dot{e}+c_{i} e-L_{i} \exp \left[-k_{i}\left(t-t_{i}\right)\right] \\
\vdots \\
s_{N}=\dot{e}+c_{N} e-L_{N} \exp \left[-k_{N}\left(t-t_{N}\right)\right]
\end{gathered}
$$

Then, we design the corresponding control law $u_{1}, u_{2}, \ldots$, $u_{i}, \ldots, u_{N}$

$$
\begin{gathered}
u_{1}=-\frac{\left[-F_{1} \dot{x}+c_{1} \dot{x}-\dot{f}_{1}+R_{1}+\left(\varepsilon_{1}+D\right) \operatorname{sign}\left(s_{1}\right)\right]}{G_{1}} \\
u_{2}=-\frac{\left[-F_{2} \dot{x}+c_{2} \dot{x}-\dot{f}_{2}+R_{2}+\left(\varepsilon_{2}+D\right) \operatorname{sign}\left(s_{2}\right)\right]}{G_{2}} \\
\vdots \\
u_{i}=-\frac{\left[-F_{i} \dot{x}+c_{i} \dot{x}-\dot{f}_{i}+R_{i}+\left(\varepsilon_{i}+D\right) \operatorname{sign}\left(s_{i}\right)\right]}{G_{i}} \\
\vdots \\
u_{N} \quad \\
=-\frac{\left[-F_{N} \dot{x}+c_{N} \dot{x}-\dot{f}_{N}+R_{N}+\left(\varepsilon_{N}+D\right) \operatorname{sign}\left(s_{N}\right)\right]}{G_{N}}
\end{gathered}
$$

where $\dot{f}_{i}$ is short for the derivative of $f_{i}(t)=L_{i} \exp \left[-k_{i}\left(t-t_{i}\right)\right]$ and $L_{i}=\left[\dot{e}\left(t_{i}\right)+c_{i} e\left(t_{i}\right)\right], R_{i}=-\ddot{x}_{c}-c_{i} \dot{x}_{c}$. Additionally, the time $0=t_{1}<t_{2}<\cdots<t_{i}<\cdots<t_{N}$ represents switching instants. The other parameters are the same as mentioned before.

Therefore, the controller for a multiple model control system is expressed as follows:

$$
u(t)= \begin{cases}u_{1}(t)=-\frac{\left[-F_{1} \dot{x}+c_{1} \dot{x}-\dot{f}_{1}+R_{1}+\left(\varepsilon_{1}+D\right) \operatorname{sign}\left(s_{1}\right)\right]}{G_{1}}, & \text { if } x_{1}^{\text {initial }} \leq x<x_{1}^{\text {terminal }} \\ u_{2}(t)=-\frac{\left[-F_{2} \dot{x}+c_{2} \dot{x}-\dot{f}_{2}+R_{2}+\left(\varepsilon_{2}+D\right) \operatorname{sign}\left(s_{2}\right)\right]}{G_{2}}, & \text { if } x_{2}^{\text {initial }} \leq x<x_{2}^{\text {terminal }} \\ \vdots & \\ u_{i}(t)=-\frac{\left[-F_{i} \dot{x}+c_{i} \dot{x}-\dot{f}_{i}+R_{i}+\left(\varepsilon_{i}+D\right) \operatorname{sign}\left(s_{i}\right)\right]}{G_{i}}, & \text { if } x_{i}^{\text {initial }} \leq x<x_{i}^{\text {terminal }} \\ \vdots & \\ u_{N}(t)=-\frac{\left[-F_{N} \dot{x}+c_{N} \dot{x}-\dot{f}_{N}+R_{N}+\left(\varepsilon_{N}+D\right) \operatorname{sign}\left(s_{N}\right)\right]}{G_{N}}, & \text { if } x_{N}^{\text {initial }} \leq x<x_{N}^{\text {terminal }}\end{cases}
$$




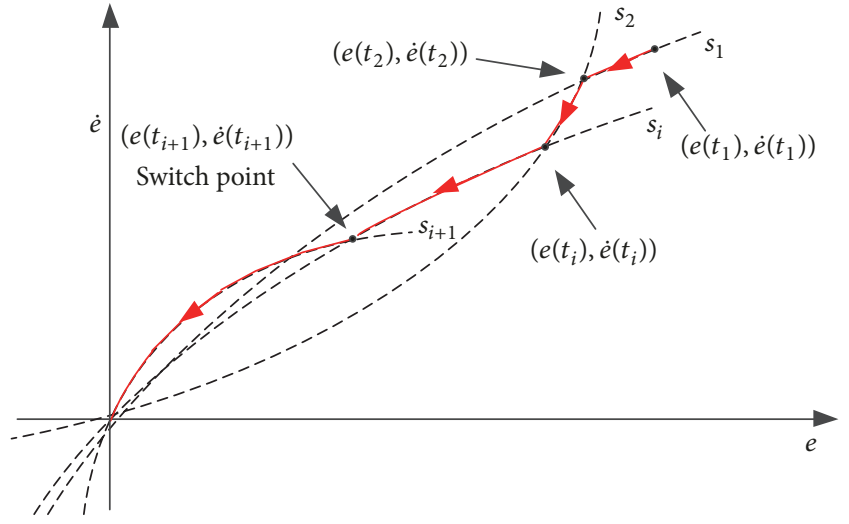

FIGURE 1: Phase trajectory of the state variable.

The state variable moves like Figure 1 shows.

The state variable moves along sliding surfaces with the order $s_{1} \longrightarrow s_{2} \longrightarrow \ldots \longrightarrow s_{i} \longrightarrow s_{i+1}$. Suppose that the initial state variable is $\left(e\left(t_{1}\right), \dot{e}\left(t_{1}\right)\right)$ and moves along the sliding surface $s_{1}$. When the state $x$ satisfies $x_{2}^{\text {initial }} \leq x<$ $x_{2}^{\text {terminal }}$, submodel $M_{1}$ switches to $M_{2}$ at the switching instant $t_{2}$, and the state variable moves to the next sliding surface $s_{2}$. By parity of reasoning, with the submodel switching from $M_{1}$ to $M_{i+1}$, the state variable moves along corresponding sliding surfaces from $s_{1}$ to $s_{i+1}$ and until the equilibrium point.

3.2.1. Lyapunov Stability Analysis. For each model in 2, the Lyapunov function is defined as $V_{i}=(1 / 2) s_{i}{ }^{2}$ and the process of proving the Lyapunov stability is the same as Section 3.1.1. We can obtain the result $\dot{V}_{i}<0$, which means the multimodel closed-loop control system based on GSMC is globally stable.

3.2.2. Finite Time Convergence Analysis. Suppose that $t_{i}$ and $t_{i+1}$ are the switching instants where surface switches from $s_{i-1}$ to $s_{i}$ and $s_{i}$ to $s_{i+1}$, respectively. Thus, the time state variable moving along the sliding surface $s_{i}$ to switching instant is $t_{i, i+1}=t_{i+1}-t_{i}$. According to (11), the state variable at switching instant $t_{i+1}$ is expressed as

$$
\begin{aligned}
e\left(t_{i+1}\right)= & \frac{L_{i}}{c_{i}-k_{i}} \exp \left(-k_{i}\left(t_{i+1}-t_{i}\right)\right) \\
& +m_{i} \exp \left(-c_{i}\left(t_{i+1}-t_{i}\right)\right)
\end{aligned}
$$

Let $A_{i}=L_{i} /\left(c_{i}-k_{i}\right), B_{i}=m_{i}, D_{i}=e\left(t_{i+1}\right)$; the equation is transformed to

$$
D_{i}=A_{i} e^{-k_{i}\left(t_{i+1}-t_{i}\right)}+B_{i} e^{-c_{i}\left(t_{i+1}-t_{i}\right)}
$$

Furthermore, let $D_{i}=E_{i} e^{-c_{i}\left(t_{i+1}-t_{i}\right)}$, in which $E_{i}$ is an undetermined parameter. Then, substitute it into (17)

$$
E_{i} e^{-c_{i}\left(t_{i+1}-t_{i}\right)}=A_{i} e^{-k_{i}\left(t_{i+1}-t_{i}\right)}+B_{i} e^{-c_{i}\left(t_{i+1}-t_{i}\right)}
$$

From (12) we can easily obtain that the time $t_{i, i+1}$ is

$$
t_{i, i+1}=\frac{1}{k_{i}-c_{i}} \ln \frac{A_{i}}{E_{i}-B_{i}}
$$

Now substitute (19) into $D_{i}=E_{i} e^{-c_{i}\left(t_{i+1}-t_{i}\right)}$ :

$$
\begin{aligned}
D_{i} & =E_{i} e^{-c_{i}\left(\left(1 /\left(k_{i}-c_{i}\right)\right) \ln \left(A_{i} /\left(E_{i}-B_{i}\right)\right)\right)} \\
& =E_{i} e^{\ln \left(A_{i} /\left(D_{i}-B_{i}\right)\right)^{c_{i} /\left(c_{i}-k_{i}\right)}}=E_{i}\left(\frac{A_{i}}{E_{i}-B_{i}}\right)^{c_{i} /\left(c_{i}-k_{i}\right)}
\end{aligned}
$$

Thus, the parameter $E_{i}$ satisfies the following equation:

$$
e\left(t_{i+1}\right)^{c_{i}-k_{i}} E_{i}^{k_{i}-c_{i}}\left(E_{i}-B_{i}\right)^{c_{i}}=A_{i}^{c_{i}}
$$

Obviously, the solution of (21) exists.

Similarly, the time that the state variable moves along other sliding surfaces can be calculated. Suppose that the last sliding surface is $s_{N}$ and its finite convergence time is $t_{N}=\left(1 /\left(k_{N}-c_{N}\right)\right) \ln \left(L_{N} / m_{N}\left(k_{N}-c_{N}\right)\right)$. Thus, the finite convergence time for the multimodel control is expressed as

$$
T_{c}=t_{N}+\sum_{i=1}^{N-1} \frac{1}{k_{i}-c_{i}} \ln \frac{L_{i}}{\left(E_{i}-m_{i}\right)\left(c_{i}-k_{i}\right)}
$$

where $E_{i}$ satisfies (21).

Particularly, because all the sliding surfaces may not be ergodic, the real convergence time should be expressed as follows:

$$
T_{c} \leq t_{N}+\sum_{i=1}^{N-1} \frac{1}{k_{i}-c_{i}} \ln \frac{L_{i}}{\left(E_{i}-m_{i}\right)\left(c_{i}-k_{i}\right)}
$$

\section{Numerical Example and Simulation}

Numerical example will be provided in the following section to verify the control performance of proposed GSMC method.

Suppose that the nonlinear system is approximated by three second-order systems expressed as

$$
\begin{aligned}
& \text { Model A: } \ddot{x}+3 \dot{x}=5 u+0.1 \sin (2 \pi t) \\
& \text { Model B: } \ddot{x}+[25+5 \sin (t)] \dot{x} \\
& \quad=[133+10 \sin (t)] u+0.1 \sin (2 \pi t) \\
& \text { Model C: } \ddot{x}+[125+5 \cos (t)] \dot{x} \\
& \quad=[13+10 \cos (t)] u+0.1 \cos (2 \pi t)
\end{aligned}
$$

The switch instant from models A to B occurs at $2 \mathrm{~s}$ and it is $5 \mathrm{~s}$ from models $\mathrm{B}$ to $\mathrm{C}$. The initial states are $[\pi / 6,0]$ and the states at two switching instants are $\left(e_{A B}, \dot{e}_{A B}\right)=$ $(0.03,-0.2)$ and $\left(e_{B C}, \dot{e}_{B C}\right)=(0.01,0.1)$, respectively. The command signal is $x_{c}=0.5 \sin (2 \pi t)$. The parameters of the sliding surface are $c_{A}=10, k_{A}=100, c_{B}=10, k_{B}=100$, $c_{C}=0.1$, and $k_{C}=100$. The simulation results are shown in Figures 2-8.

Furthermore, we take the method and model in [29] as a comparison to show the advantage of GSMC.

The system is expressed as follows:

$$
\begin{aligned}
& \dot{x}_{1}=x_{2} \\
& \dot{x}_{2}=\theta_{1} x_{1}^{2}+\theta_{2} x_{2}^{2}+\theta_{3} x_{1} x_{2}+b u
\end{aligned}
$$




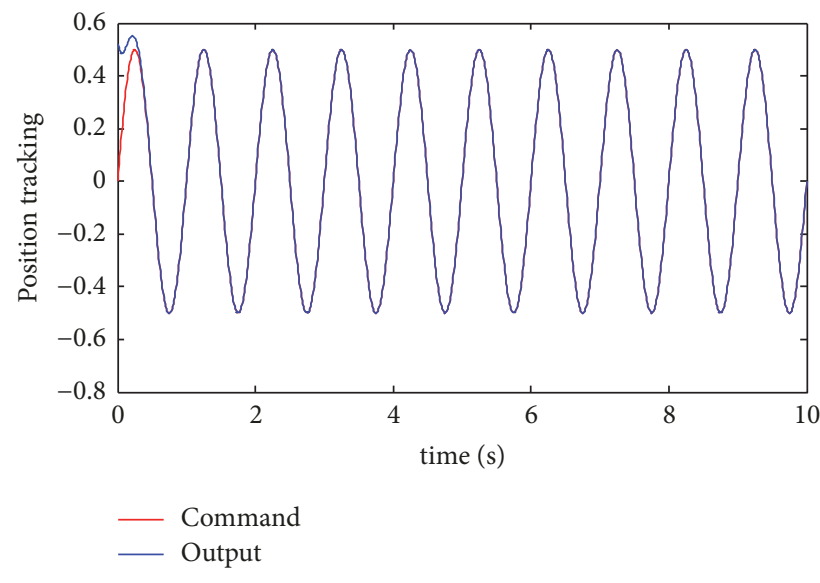

FIGURE 2: The position tracking.

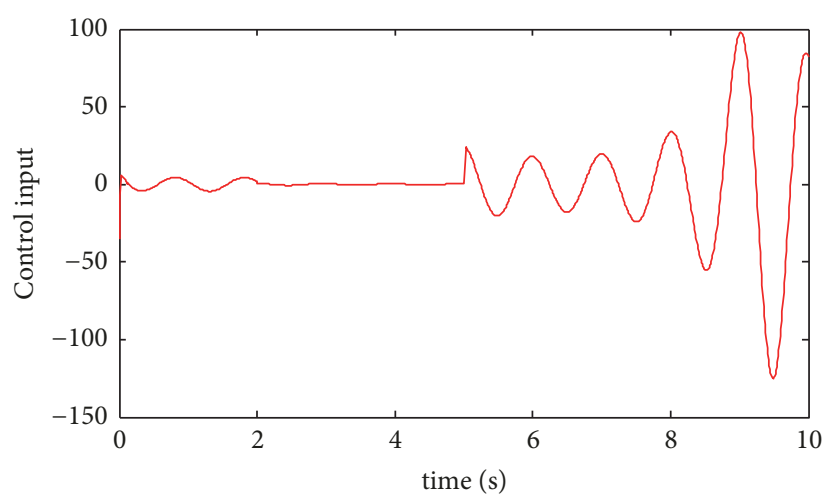

FIgURE 3: The control input.

Then, we set the switching instants and parameters of multiple models as [29] given and list them in Table 1.
Using GSMC, the controller is designed as follows:

$$
u(t)= \begin{cases}u_{1}=-\frac{\left[\theta_{1} x^{2}+\left(\theta_{2}+c_{1}+\theta_{3} x\right) \dot{x}-\dot{f}_{1}+R_{1}+\left(\varepsilon_{1}+D\right) \operatorname{sign}\left(s_{1}\right)\right]}{b}, & \text { if } t \leq 6 s \\ u_{2}=-\frac{\left[\theta_{1} x^{2}+\left(\theta_{2}+c_{2}+\theta_{3} x\right) \dot{x}-\dot{f}_{2}+R_{2}+\left(\varepsilon_{2}+D\right) \operatorname{sign}\left(s_{2}\right)\right]}{b}, & \text { if } 6<t \leq 16 s \\ u_{3}=-\frac{\left[\theta_{1} x^{2}+\left(\theta_{2}+c_{3}+\theta_{3} x\right) \dot{x}-\dot{f}_{3}+R_{3}+\left(\varepsilon_{3}+D\right) \operatorname{sign}\left(s_{3}\right)\right]}{b}, & \text { if } 16<t \leq 32 s \\ u_{4}=-\frac{\left[\theta_{1} x^{2}+\left(\theta_{2}+c_{4}+\theta_{3} x\right) \dot{x}-\dot{f}_{4}+R_{4}+\left(\varepsilon_{4}+D\right) \operatorname{sign}\left(s_{4}\right)\right]}{b}, & \text { if } t>32 s\end{cases}
$$

Then, we obtain the simulation results in Figures 9 and 10.

From Figures 2-8, the results show that the output tracks are fast and accurate. The sliding dynamic converges fast as shown in Figure 4. What is more, it converges to 0 at switching instants immediately when the saltation occurs. From the phase trajectory in Figures 5-8 we can conclude that the error state $(e, \dot{e})$ moves along the sliding surfaces to the equilibrium point. At the two switching instants, the GSMC steers $(e, \dot{e})$ to the next surface both within two steps (0.02s in simulation). Thus, the validity and effectiveness of GSMC for multiple models are verified.

Furthermore, compared with the multimodel robust control method of [29] in Figures 9 and 10, the convergence characteristic of GSMC provides fast stability at switch instants, which increases the robust to the system mutation. 


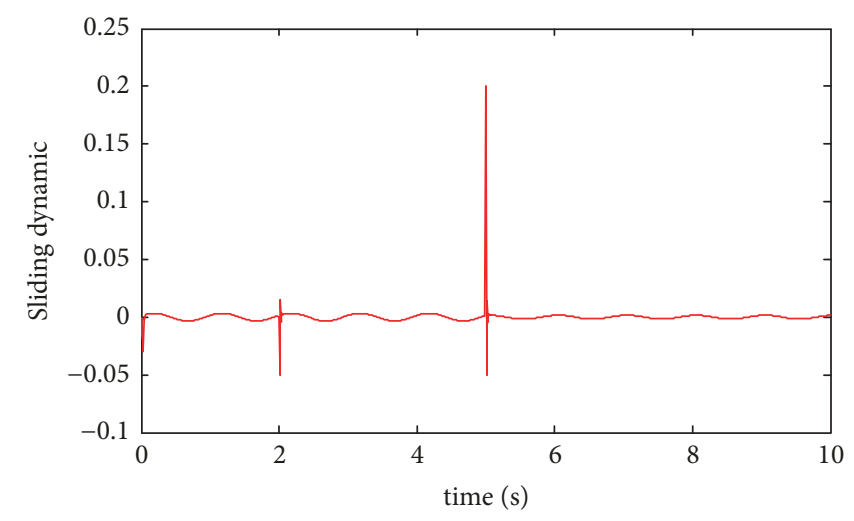

FIgURE 4: The sliding dynamic.

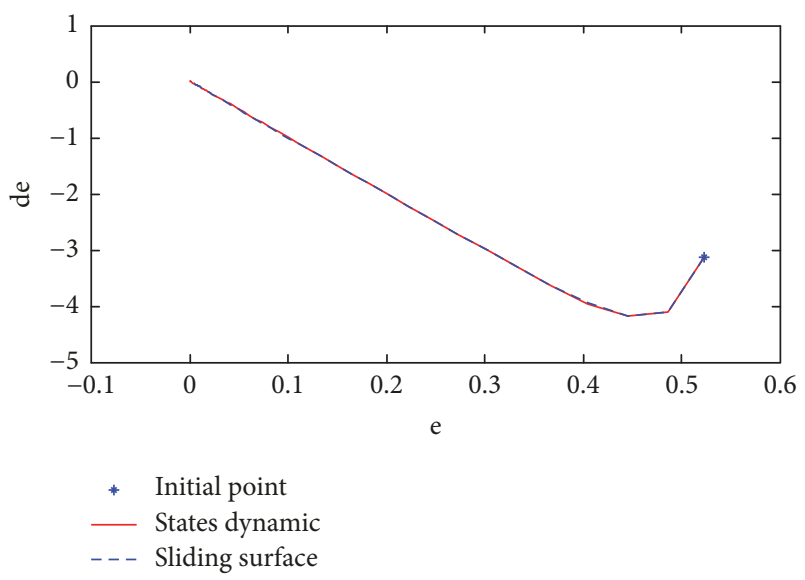

FIGURE 5: The sliding dynamic of model A.

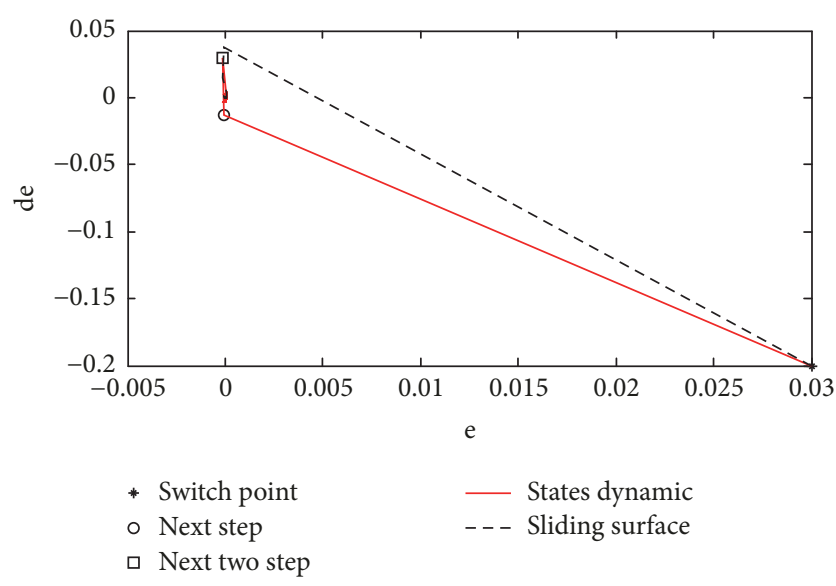

FIGURE 6: The sliding dynamic of model B.

\section{Conclusion}

In this paper, a global sliding mode control method for second-order multimodel system was proposed. The Lyapunov stability and finite time convergence for both single model and multiple model were proved.

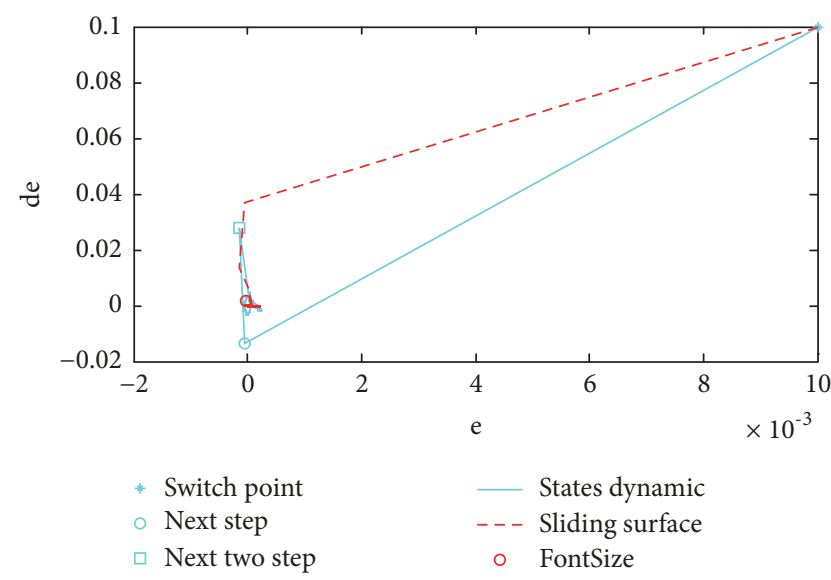

FIGURE 7: The sliding dynamic of model C.

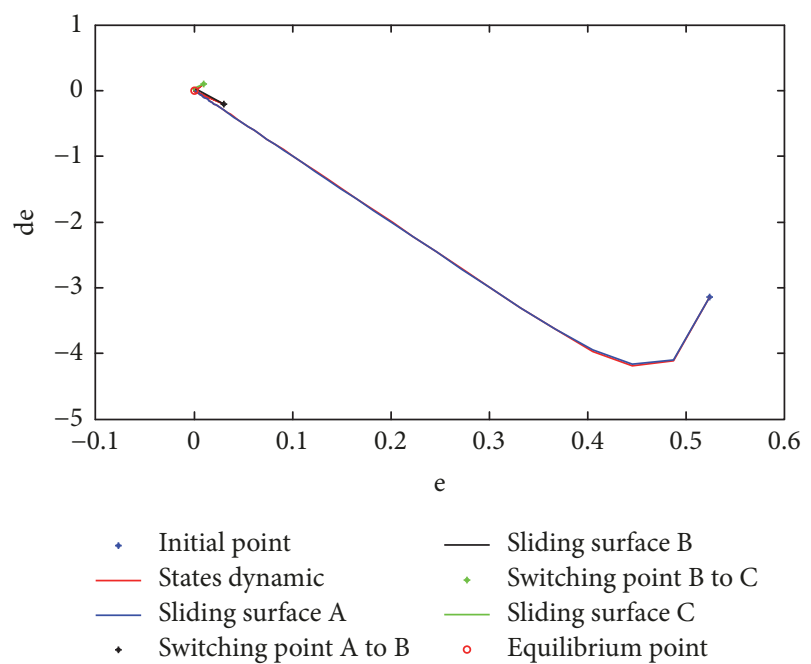

Figure 8: The phase trajectory.

Finally, the feasibility and effectiveness of the proposed control method were verified through a numerical simulation. The simulation results conclude that the control law based on GSMC is effective for the multimodel control 


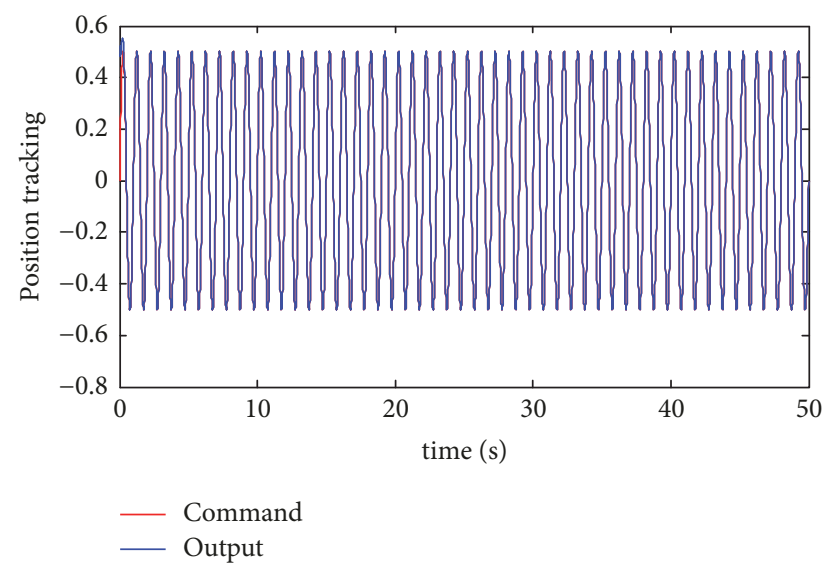

FIGURE 9: The position tracking.

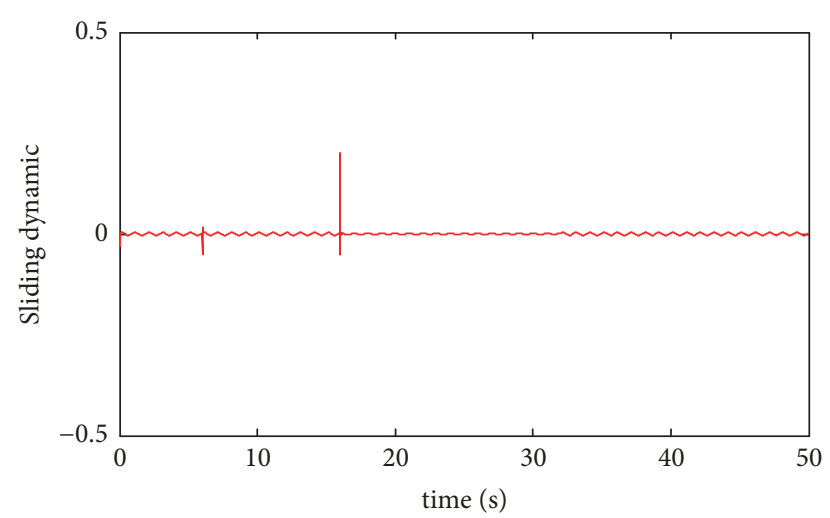

FIgUre 10: The sliding dynamic.

TABLE 1: The parameters of models in [29].

\begin{tabular}{lcccc}
\hline Switch instant & $0 \mathrm{~s}$ & $6 \mathrm{~s}$ & $16 \mathrm{~s}$ & $32 \mathrm{~s}$ \\
\hline$\theta_{1}$ & -1 & -1 & -0.5 & 0.5 \\
\hline$\theta_{2}$ & 3 & 2 & 0.2 & -2.5 \\
\hline$\theta_{3}$ & 1 & 0.5 & 0.2 & -0.5 \\
\hline$b$ & 0.1 & 1 & 2 & 5 \\
\hline
\end{tabular}

problem. And also, the closed-loop control system output can track the command signal fast and accurately. Compared with another multimodel control method in [29], GSMC provides faster convergence at switching instants and promotes the global robust performance of the controller.

\section{Data Availability}

All the data used in the article is available and can be downloaded from the network address "https://pan.baidu.com/s/ 1wYaxh6qnWMwuT_jEFf 350 CWhA" or is available from the corresponding author upon request.

\section{Conflicts of Interest}

The authors declare that there are no conflicts of interest regarding the publication of this paper.

\section{Acknowledgments}

This work has been supported by the Major Program of the National Natural Science Foundation of China (no. 61627901).

\section{References}

[1] X. Lu and H. Dong, "Application of Multi-model Active Faulttolerant Sliding Mode Predictive Control in Solar Thermal Power Generation System," Zidonghua Xuebao/Acta Automatica Sinica, vol. 43, no. 7, pp. 1241-1247, 2017.

[2] K. Xue and Y. Li, "A Multi-model Modeling Approach to MIMO Nonlinear Systems," ACTA Electronica Sinica, vol. 33, no. 1, 2005.

[3] Y. Fang, D. Chai, D. Mao, and L. Zhang, "Status and development trend of the guidance and control for air-breathing hypersonic vehicle," Hangkong Xuebao/Acta Aeronautica et Astronautica Sinica, vol. 35, no. 7, pp. 1776-1786, 2014.

[4] H. Duan and P. Li, "Progress in control approaches for hypersonic vehicle," Science China Technological Sciences, vol. 55, no. 10, pp. 2965-2970, 2012.

[5] B. Xu and Z. K. Shi, "An overview on flight dynamics and control approaches for hypersonic vehicles," Science China Information Sciences, 2015.

[6] L. Wu and B. Meng, "Revies on The Control of Hypersonic Flight Vehicles," Advance in Mechanics, vol. 39, no. 6, pp. 756765, 2009.

[7] C.-Y. Sun, C.-X. Mu, and Y. Yu, "Some control problems for near space hypersonic vehicles," Zidonghua Xuebao/Acta Automatica Sinica, vol. 39, no. 11, pp. 1901-1913, 2013.

[8] E. J. Cui, "Research Statutes, Development Trends and Key Technical Problems of Near Space Flying Vehicles," Advance in Mechanics, vol. 36, pp. 658-673, 2009.

[9] L. Huang, Z.-S. Duan, and J.-Y. Yang, "Challenges of control science in near space hypersonic aircrafts," Kongzhi Lilun Yu Yingyong/Control Theory and Applications, vol. 28, no. 10, pp. 1496-1505, 2011.

[10] M.-G. Gan, M. Zhang, H.-X. Ma, and J. Chen, "Adaptive control of a servo system based on multiple models," Asian Journal of Control, vol. 18, no. 2, pp. 652-663, 2016.

[11] R. A. DeCarlo, S. H. Zak, and G. P. Matthews, "Variable structure control of nonlinear multivariable systems: a tutorial," Proceedings of the IEEE, vol. 76, no. 3, pp. 212-232, 1988.

[12] A. E. Ariffin and N. Munro, "Robust control analysis of a gasturbine aeroengine," IEEE Transactions on Control Systems Technology, vol. 5, no. 2, pp. 178-188, 1997.

[13] G. Bartolini, A. Ferrara, E. Usai, and V. I. Utkin, "On multiinput chattering-free second-order sliding mode control," IEEE Transactions on Automatic Control, vol. 45, no. 9, pp. 1711-1717, 2000.

[14] A. Levant, "Principles of 2-sliding mode design," Automatica, vol. 43, no. 4, pp. 576-586, 2007.

[15] I. Boiko, L. Fridman, and M. I. Castellanos, "Analysis of secondorder sliding-mode algorithms in the frequency domain," Institute of Electrical and Electronics Engineers Transactions on Automatic Control, vol. 49, no. 6, pp. 946-950, 2004.

[16] C. Edwards and S. K. Spurgeon, Sliding Mode Control: Theory And Applications, Francis Systems and Control Book Series, Taylor \& Francis, Taylor, 1998. 
[17] V. I. Utkin, Sliding Modes in Control Optimization, Springer, Berlin, Germany, 1991.

[18] B. W. Gao, The Theory And Design Method of Variable Structure Control, Science Press, Beijing, China, 1998.

[19] A. Levant, "Chattering analysis," IEEE Transactions on Automatic Control, vol. 55, no. 6, pp. 1380-1389, 2010.

[20] G. C. Goodwin, D. J. Hill, and M. Palaniswami, "A perspective on convergence of adaptive control algorithms," Automatica, vol. 20, no. 5, pp. 519-531, 1984.

[21] T. L. Tai and Y. S. Lu, "Global sliding mode control with chatter alleviation for robust eigenvalue assignment," Proceedings of the Institution of Mechanical Engineers, Part I: Journal of Systems and Control Engineering, vol. 220, no. 7, pp. 573-584, 2006.

[22] S.-B. Choi, C.-C. Cheong, and D.-W. Park, "Moving switching surfaces for robust control of second-order variable structure systems," International Journal of Control, vol. 58, no. 1, pp. 229245, 1993.

[23] T.-H. Chang and Y. Hurmuzlu, "Sliding control without reaching phase and its application to bipedal locomotion," Journal of Dynamic Systems, Measurement, and Control, vol. 115, no. 3, pp. 447-455, 1993.

[24] S. Y. Lu and S. J. Chen, "Design of a global sliding mode controller for robot manipulator with robust tracking capability," Proceedings of the National Science Council, Republic of China Part A: Physical Science and Engineering, vol. 18, pp. 463-476, 1994.

[25] Y.-S. Lu and J.-S. Chen, "Design of a global sliding-mode controller for a motor drive with bounded control," International Journal of Control, vol. 62, no. 5, pp. 1001-1019, 1995.

[26] J.-Y. Lin, Y.-S. Lu, and J.-S. Chen, "A global sliding-mode control scheme with adjustable robustness for a linear variable reluctance motor," JSME International Journal Series C Mechanical Systems, Machine Elements and Manufacturing, vol. 45, no. 1, pp. 215-225, 2002.

[27] H. S. Choi, Y.-H. Park, Y. Cho, and M. Lee, "Global sliding-mode control. Improved design for a brushless DC motor," IEEE Control Systems Magazine, vol. 21, no. 3, pp. 27-35, 2001.

[28] K.-K. Shyu and H.-J. Shieh, "A new switching surface slidingmode speed control for induction motor drive systems," IEEE Transactions on Power Electronics, vol. 11, no. 4, pp. 660-667, 1996.

[29] L. Shao, H.-M. Lei, and Z.-L. Tang, "Multiple-model composite control based on LQ/VSC," Xi Tong Gong Cheng Yu Dian Zi Ji Shu, vol. 31, no. 5, pp. 1194-1197, 2009.

[30] J. Yan, F. Y. Yu, and Y. H. Fan, The Control Technology of Airbreathing Hypersonic Vehicle, Northwestern Polytechnic University Press, Xi’an, China, 2015.

[31] V. F. Zaitsev and A. D. Polyanin, Handbook of Exact Solutions for Ordinary Differential Equations, CRC Press, 2002. 


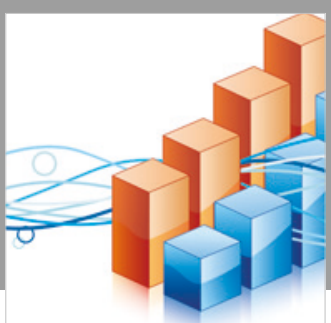

Advances in

Operations Research

\section{-n-m}
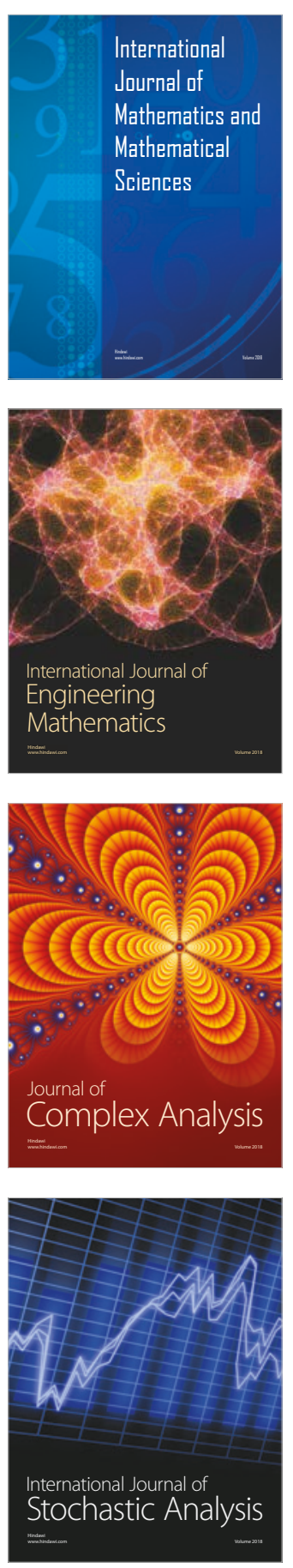
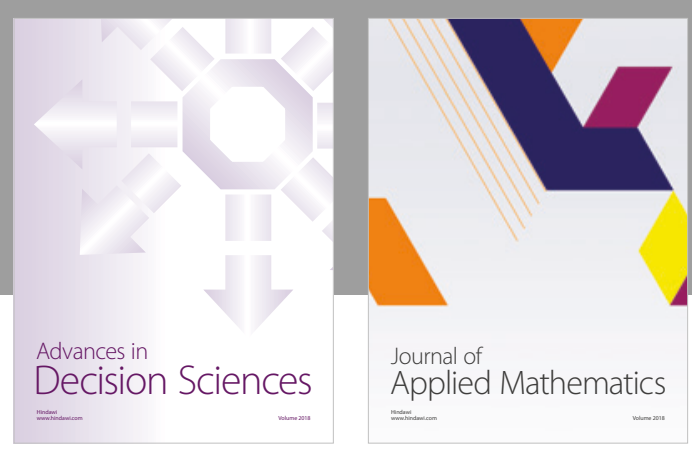

Journal of

Applied Mathematics
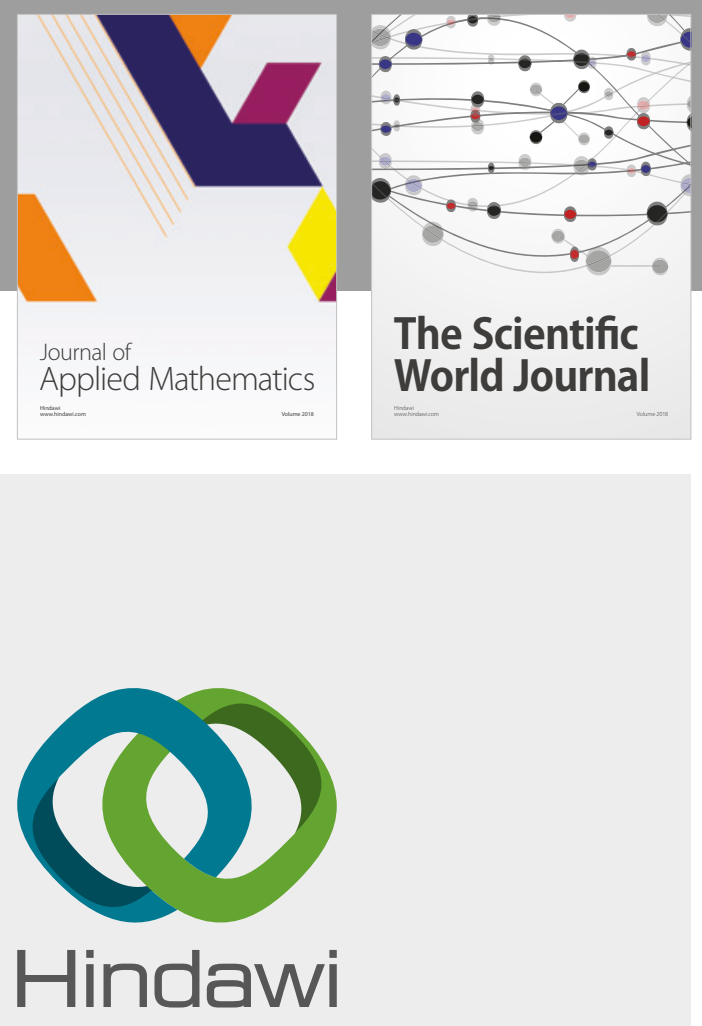

Submit your manuscripts at

www.hindawi.com

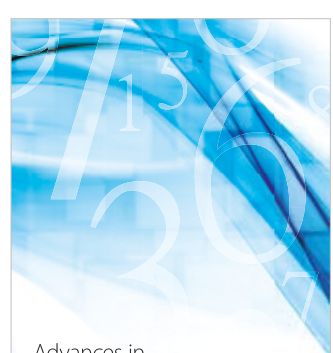

Advances in
Numerical Analysis
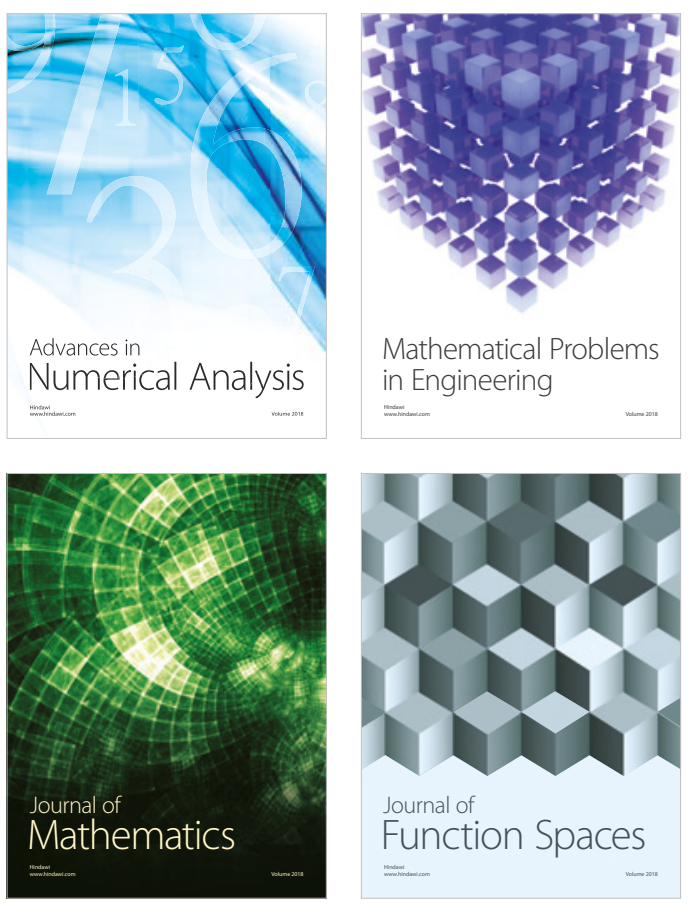

Mathematical Problems in Engineering

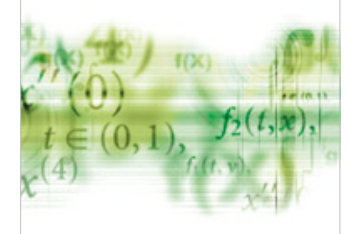

International Journal of

Differential Equations

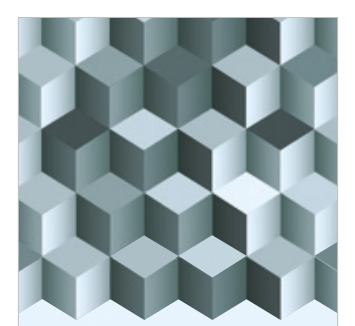

Journal of

Function Spaces

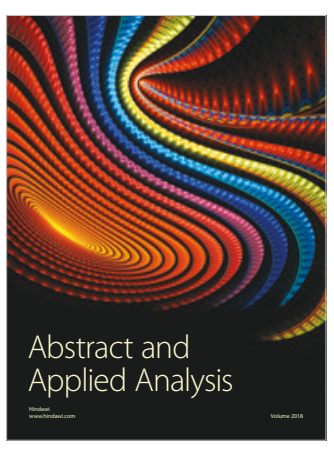

The Scientific

World Journal

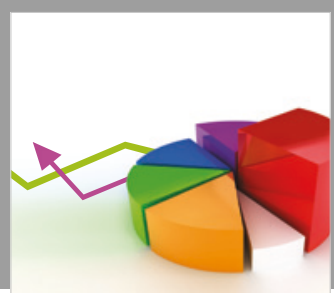

Journal of

Probability and Statistics
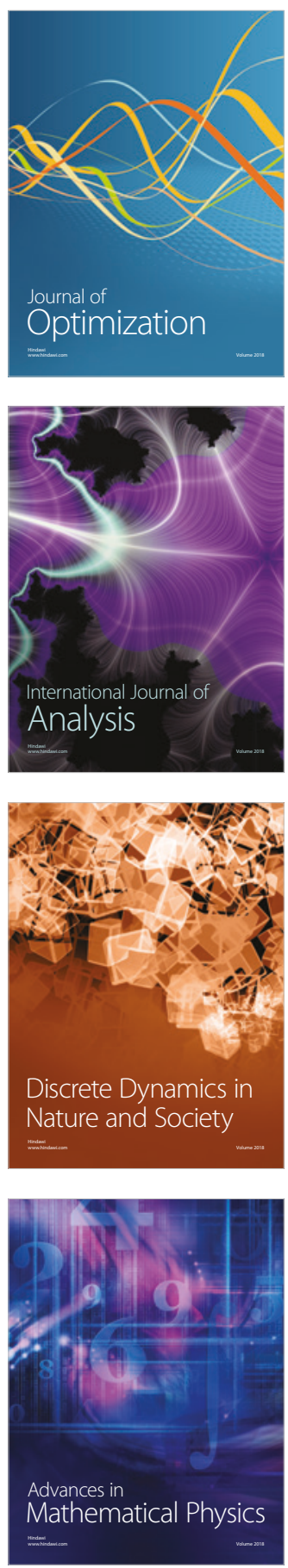